\title{
Analytical investigations of ground modifications assisting the detection of a buried object
}

\author{
Constantinos A. Valagiannopoulos*, Nikolaos L. Tsitsas ${ }^{\dagger}$, and Ari H. Sihvola ${ }^{\ddagger}$ \\ *Department of Physics, Nazarbayev University, Astana 010000, Kazakhstan \\ e-mail: konstantinos.valagiannopoulos@nu.edu.kz \\ $\dagger$ Department of Informatics, Aristotle University of Thessaloniki, Thessaloniki 54124, Greece \\ e-mail: ntsitsas@csd.auth.gr \\ $\ddagger$ Department of Radio Science and Engineering, Aalto University, Aalto 00076, Finland \\ e-mail: ari.sihvola@aalto.fi
}

\begin{abstract}
A ground-penetrating radar (GPR) antenna excites a perfectly electric conducting inclusion buried inside the ground. The scattering problem is solved semi-analytically via integral equation techniques. The permittivity and thickness of a superstrate deposited atop the ground are determined such that the detectability of the inclusion is significantly increased. Results from numerical simulations are presented exhibiting the effectiveness of the approach. Emphasis is given on the effects that the shape of the buried inclusion has on the scattered field.
\end{abstract}

\section{INTRODUCTION}

Important applications like detection of mines [1], mineral deposits [2], and unexploded ordnances [3] as well as scattering by buried pipes [4] motivate the analysis of inverse scattering problems of locating buried inclusions inside the earth. The methodologies widely used for the solution of such problems employ primary fields generated by ground-penetrating radars (GPRs) [5], [6] and rely on the establishment of efficient algorithms concerning the processing of the scattering data in order for the inclusion's location to be revealed. Crucial characteristics of the related GPR approaches are the number of utilized frequencies and the type of the primary field (pulse or harmonic wave); see indicatively [7] and [8].

In this work, we follow a new route aiming at increasing the detectability of a buried inclusion and, hence, preparing the ground for a more effective implementation of scattering processing algorithms (like the ones of [5]- [8]). More precisely, we modify the structure of the considered configuration by depositing on the lossy ground a suitable passive superstrate layer assisting both the primary field's penetration in the ground as well as the buried inclusion's scattering response in the air region. The main purpose lies in the determination of the permittivity and thickness of the superstrate such that the detectability of a cylindrical perfectly electric conducting (PEC) buried inclusion is increased.

The scattering problem is solved by means of semianalytical integral equation techniques. In particular, the associated boundary value problem is reformulated via a Fredholm integral equation for the current flowing on the surface of the PEC inclusion. This integral equation is solved by a semianalytical methodology providing high numerical stability and controllable accuracy. Subsequently, we show that the optimal superstrate layer's parameters are not significantly affected by the size and depth of the inclusion and therefore it is actually the inclusion-free configuration that primarily dictates the optimal parameters determination.

Several numerical simulations results are presented demonstrating the possibility of amplifying significantly the scattered far-field response of the inclusion by covering the ground with a suitable superstrate layer. A major objective of this work concerns the investigation of the effects on the amplification of the scattered field due to the different considered shapes of the buried inclusion.

An $\exp (+j 2 \pi f t)$ time dependence is assumed and suppressed throughout the analysis, where $f$ is the operational frequency of the single-frequency GPR.

\section{INTEGRAL EQUATION ANALYSIS OF THE SCATTERING PROBLEM}

A PEC cylindrical inclusion of radius $a$ is buried at a distance $d$ inside the ground which is characterized by relative complex permittivity $\varepsilon_{r 1}$; see Fig. 1 . The buried PEC cylinder is electrically small, i.e. $k_{0} a \ll 1$, where $k_{0}=\frac{2 \pi f}{c}$ is the free-space wavenumber with $c=\frac{1}{\sqrt{\varepsilon_{0} \mu_{0}}}$ the speed of light (in vacuum with permittivity $\varepsilon_{0}$ and permeability $\mu_{0}$ ).

The main purpose concerns the selection of the thickness $h$ and the complex relative permittivity $\varepsilon_{r 2}$ of a thin layer $\left(k_{0} h<\right.$ 1 ), deposited atop the ground, such that the detection of the inclusion's location becomes more feasible. The primary field is due to a moving GPR line-source carrying electric current $I$ and located in free space (region $\# 0)$ at $(x, y)=(\chi, d+h)$. Its $z$-component is given by the Fourier integral [9]

$$
\begin{aligned}
E_{0, i n c}(x, y)= & -\frac{j I k_{0} \zeta_{0}}{4 \pi} \times \\
& \int_{-\infty}^{+\infty} \frac{e^{-g_{0}(\beta)|y-d-h|}}{g_{0}(\beta)} e^{-j \beta(x-\chi)} d \beta,
\end{aligned}
$$

where $\zeta_{0}=\sqrt{\mu_{0} / \varepsilon_{0}}$ is the free-space impedance and $g_{0}(\beta)=$ $\sqrt{\beta^{2}-k_{0}^{2}}$. 


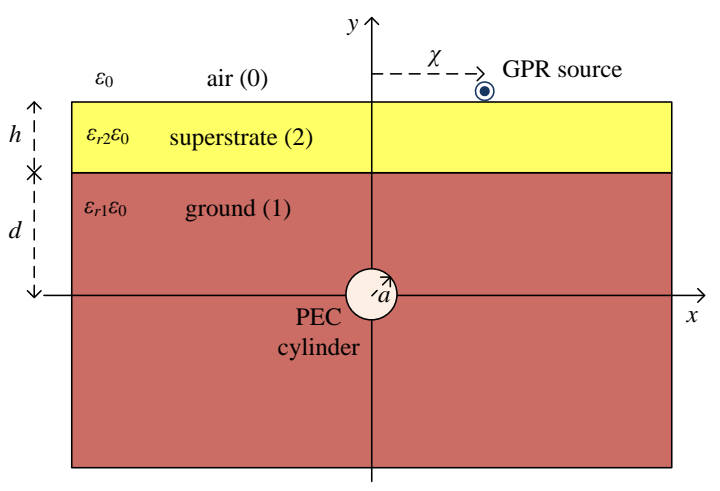

Fig. 1. A dielectric layer of suitable width $h$ and permittivity $\varepsilon_{r 2}$ is deposited on the ground (permittivity $\varepsilon_{r 1}$ ) to increase the detectability of a buried PEC inclusion at depth $d$. The inclusion has electrically small size $2 a$ and is excited by an electric line source of current $I$ positioned on the vacuum-superstrate surface at horizontal distance $\chi$ from the inclusion.

The $z$-component of the background field into the ground (region \#1) in the absence of the inclusion takes the form

$$
E_{1, b a c k}(x, y)=\int_{-\infty}^{+\infty} \gamma_{b a c k}(\beta) e^{g_{1}(\beta) y} e^{-j \beta(x-\chi)} d \beta,
$$

where

$$
\begin{aligned}
& \gamma_{\text {back }}(\beta)=\frac{j I k_{0} \zeta_{0}}{\pi} g_{2}(\beta) e^{-g_{1}(\beta) d} \times \\
& {\left[e^{-g_{2}(\beta) h}\left(g_{2}(\beta)-g_{0}(\beta)\right)\left(g_{2}(\beta)-g_{1}(\beta)\right)\right.} \\
& \left.-e^{g_{2}(\beta) h}\left(g_{2}(\beta)+g_{0}(\beta)\right)\left(g_{2}(\beta)+g_{1}(\beta)\right)\right]^{-1},
\end{aligned}
$$

with $g_{1}(\beta)=\sqrt{\beta^{2}-k_{0}^{2} \varepsilon_{r 1}}$ and $g_{2}(\beta)=\sqrt{\beta^{2}-k_{0}^{2} \varepsilon_{r 2}}$.

The Green's function, corresponding to a line source excitation at $(X, Y)$ in region $\# 1$ and observation vector $(x, y)$ in region $\# 1$, is comprised of a primary (singular) term $G_{1}^{p r}$ and a secondary (smooth) term $G_{1}^{s e c}$, respectively, given by

$$
\begin{aligned}
G_{1}^{p r}(x, y, X, Y) & =-\frac{j}{4} H_{0}^{(2)}\left(k_{0} \sqrt{(x-X)^{2}+(y-Y)^{2}}\right), \\
G_{1}^{s e c}(x, y, X, Y) & =\int_{-\infty}^{+\infty} \gamma_{1}^{s e c}(\beta) e^{g_{1}(\beta)(y+Y)} e^{-j \beta(x-X)} d \beta,
\end{aligned}
$$

where $H_{0}^{(2)}$ denotes the zeroth order and second kind cylindrical Hankel function, while

$$
\begin{aligned}
& \gamma_{1}^{s e c}(\beta)=e^{-2 g_{1}(\beta) d}\left[\cosh \left(g_{2}(\beta) h\right)\left(g_{1}(\beta)-g_{0}(\beta)\right) g_{2}(\beta)\right. \\
& \left.+\left(g_{0}(\beta) g_{1}(\beta)-g_{2}^{2}(\beta)\right) \sinh \left(g_{2}(\beta) h\right)\right] \times \\
& {\left[4 \pi g_{1}(\beta)\right]^{-1}\left[\cosh \left(g_{2}(\beta) h\right)\left(g_{1}(\beta)+g_{0}(\beta)\right) g_{2}(\beta)+\right.} \\
& \left.\left(g_{0}(\beta) g_{1}(\beta)+g_{2}^{2}(\beta)\right) \sinh \left(g_{2}(\beta) h\right)\right]^{-1} .
\end{aligned}
$$

For $(x, y)$ in region $\# 0$, the Green's function takes the form

$$
G_{0}(x, y, X, Y)=\int_{-\infty}^{+\infty} \gamma_{0}(\beta) e^{-g_{0}(\beta)(y-Y)} e^{-j \beta(x-X)} d \beta,
$$

where

$$
\begin{aligned}
& \gamma_{0}(\beta)=\left[\pi g_{0}(\beta)\right]^{-1} e^{g_{0}(\beta)(d+h)-g_{1}(\beta) d} g_{0}(\beta) g_{2}(\beta) \times \\
& {\left[e^{g_{2}(\beta) h}\left(g_{0}(\beta)+g_{2}(\beta)\right)\left(g_{1}(\beta)+g_{2}(\beta)\right)\right.} \\
& \left.-e^{-g_{2}(\beta) h}\left(g_{0}(\beta)-g_{2}(\beta)\right)\left(g_{1}(\beta)-g_{2}(\beta)\right)\right]^{-1} .
\end{aligned}
$$

The generated scattered field in region $\# 1$, due to the presence of the PEC cylinder, has the integral representation

$$
\begin{aligned}
E_{1, s c}(x, y) & =-j k_{0} \zeta_{0} \times \\
& \int_{C} K(l)\left[\begin{array}{c}
G_{1}^{p r}(x, y, X(l), Y(l)) \\
+G_{1}^{s e c}(x, y, X(l), Y(l))
\end{array}\right] d l,
\end{aligned}
$$

where $K(l)$ is the current along the cylinder's boundary $C$.

For an electrically small cylinder, we can make the thinwire approximation [9] and infer that the (unknown) current $K$ is constant along $C$. In this way, the scattered field $E_{1, s c}$ at $(x, y)=(0,0)$ is approximated by

$$
\begin{aligned}
E_{1, s c}(0,0) \cong & -\frac{\pi k_{0} a \zeta_{0} K}{2} H_{0}^{(2)}\left(k_{0} a \sqrt{\varepsilon_{r 1}}\right) \\
& -2 \pi j k_{0} a \zeta_{0} K G_{1}^{s e c}(0,0,0,0) .
\end{aligned}
$$

By applying the PEC boundary condition at the center of the cylinder

$$
E_{1, b a c k}(0,0)+E_{1, s c}(0,0)=0,
$$

we get

$$
K=\frac{j}{2 \pi k_{0} a \zeta_{0}} \frac{E_{1, \text { back }}(0,0)}{\frac{j}{4} H_{0}^{(2)}\left(k_{0} a \sqrt{\varepsilon_{r 1}}\right)-G_{1}^{s e c}(0,0,0,0)},
$$

and then the scattered field in region $\# 0$ is approximated by

$$
\begin{aligned}
E_{0, s c}(x, y) & \cong-2 \pi j k_{0} a \zeta_{0} K G_{0}(x, y, 0,0), \\
& =-2 \pi j k_{0} a \zeta_{0} K \int_{-\infty}^{+\infty} \gamma_{0}(\beta) e^{-g_{0}(\beta) y-j \beta x} d \beta .
\end{aligned}
$$

The scattered power in the upper half-plane is obtained by using the method of stationary phase [10], as follows

$P_{s c}=8 \pi^{4}\left(k_{0} a\right)^{2} k_{0} \zeta_{0}|K|^{2} \int_{0}^{\pi}\left|\gamma_{0}\left(k_{0} \cos \phi\right)\right|^{2} \sin ^{2} \phi d \phi$.

\section{Determination of the Optimal Superstrate}

Applications related to scattering by buried objects in the ground usually employ frequencies $f$ in the UHF range from $500 \mathrm{MHz}$ to $1 \mathrm{GHz}$. The variations of the complex permittivity $\varepsilon_{r 1}$ in this frequency range are negligible for all cases of very dry, medium dry or wet ground, namely

1) very dry ground: $\varepsilon_{r 1}^{d r y} \cong 3-j 0.05\left(\frac{f}{10^{6}}\right)^{-0.4} \cong 3-$ j0.0036,

2) medium dry ground: $\varepsilon_{r 1}^{\text {medium }} \cong 15-j 0.1\left(\frac{f}{10^{6}}\right)^{0.25} \cong$ $15-j 0.52$,

3) wet ground: $\varepsilon_{r 1}^{w e t} \cong 30-j 3.2$,

with $f$ measured in $\mathrm{Hz}$ in 1) and 2). The validity of these approximations is justified in [11]. 
The aim is that the deposited passive superstrate with optimally selected permittivity $\varepsilon_{r 2}$ and thickness $h$ will "unlock" the ground so that this superstrate will make the configuration of the ground and the superstrate to have effective properties which will increase the scattered power from the hidden target.

The determined superstrate is expected to offer these "unlocking" characteristics for any type of inclusion by substantially amplifying its scattered field. In particular, the superstrate should enhance the transmission of the incident field into the ground so that the PEC cylinder is maximally excited and simultaneously it should effectively transmit the scattered field from the inclusion back to free-space. These two objectives are equivalent for the considered reciprocal superstrate.

In order to find layers which conform to these specifications, we consider the inclusion-free structure excited by a normally incident plane wave. The inclusion-free consideration is made in order to obtain a solution that can work for every inclusion regardless of its position or size. Plane wave incidence is considered for the sake of simplicity and in order to obtain results independent of the line-source's position. Our aim is to maximize the transmission coefficient $T_{1}$ of the incident field into the ground and the transmission coefficient $T_{0}$ of the reflected field into free space. These two coefficients are proportional to each other (due to reciprocity) and defined as

$$
\begin{aligned}
& \frac{T_{0}}{\sqrt{\varepsilon_{r 1}}}=T_{1} e^{-j k_{0} h} \\
& =4 \sqrt{\varepsilon_{r 2}}\left[e^{j k_{0} h \sqrt{\varepsilon_{r 2}}}\left(1+\sqrt{\varepsilon_{r 2}}\right)\left(\sqrt{\varepsilon_{r 1}}+\sqrt{\varepsilon_{r 2}}\right)\right. \\
& \left.-e^{-j k_{0} h \sqrt{\varepsilon_{r 2}}}\left(1-\sqrt{\varepsilon_{r 2}}\right)\left(\sqrt{\varepsilon_{r 1}}-\sqrt{\varepsilon_{r 2}}\right)\right]^{-1} \equiv T .
\end{aligned}
$$

For a fixed frequency $f$ and ground's permittivity $\varepsilon_{r 1}$, the quantity $|T|$ to be maximized is a sole function of a complex variable $\varepsilon_{r 2}$ and a positive real variable $k_{0} h$. Typical variations of $|T|$ on the complex plane of the layer's permittivity $\varepsilon_{r 2}$ are depicted in [11], where it is elaborated that maximal value of $|T|$ is achieved for a lossless superstrate $\left(\operatorname{Im}\left(\varepsilon_{r 2}\right)=0\right)$. Hence, we restrict the parametric space by adopting only real permittivities $\varepsilon_{r 2}$ and seek to maximize a function $|T|$ of $\frac{h}{\lambda_{0}}>0$ and $\varepsilon_{r 2}>1$. This maximization is performed systematically in [11] and the optimal values of the permittivity $\varepsilon_{r 2}$ and electric thickness $k_{0} h$ for the very dry, medium dry and wet ground are, respectively, found to be $\varepsilon_{r 2, o p t}^{d r y}=1.84, \varepsilon_{r 2, o p t}^{\text {medium }}=3.88$, and $\varepsilon_{r 2, o p t}^{\text {wet }}=5.52$ and $k_{0} h_{\text {opt }}^{\text {dry }}=0.94, k_{0} h_{\text {opt }}^{\text {medium }}=0.79$, and $k_{0} h_{\text {opt }}^{\text {wet }}=0.66$.

These optimal parameters correspond to the inclusion-free configuration and their performance needs to be tested in the complete configuration of Fig. 1 incorporating the buried inclusion. To this end, we investigate the variations (for each ground type) of the ratios $\frac{p}{p^{\prime}}$ versus $\frac{h}{\lambda_{0}}$ and $\varepsilon_{r 2}$, where

$$
p=\int_{-\mathcal{X}}^{+\mathcal{X}} P_{s c}(\chi) d \chi \quad, \quad p^{\prime}=\int_{-\mathcal{X}}^{+\mathcal{X}} P_{s c}^{\prime}(\chi) d \chi,
$$

with $P_{s c}$ the scattered power in the presence of the superstrate given by (7), $P_{s c}^{\prime}$ the corresponding power in the absence of the superstrate $\left(\varepsilon_{r 2}=1\right)$, while $\mathcal{X}$ is a suitably large distance assuring convergence of the integrals. These ratios indicate how much more detectable the inclusion becomes in the presence of the specific superstrate (for all positions of the line source).

It is found that the ratio $\frac{p}{p^{\prime}}$ attains maximum values at points close to the corresponding ones that the function $|T|$ is maximized; for details as well as depictions of these results see [11]. Moreover, these maximum values of $\frac{p}{p^{\prime}}$ are substantial and hence the pairs $\left(\varepsilon_{r 2, o p t}, k_{0} h_{o p t}\right)$, selected through the $|T|$ maximization criterion, are indeed effective in amplifying the scattering response from the inclusion. Therefore, the strategy of simplifying the configuration (inclusion-free) and the excitation (plane wave) in order to extract the optimal $\varepsilon_{r 2, o p t}$ and $k_{0} h_{o p t}$ is effective and yields satisfactory results for the full problem.

\section{Numerical Results}

The validity and performance of the developed methodology are tested by simulating certain scattering problems concerning a normally incident plane wave impinging on a buried inclusion of arbitrary shape at $d=1.8 \lambda_{0}$. Scattered field simulations are performed with COMSOL Multiphysics [12]; the consideration of the plane incident wave was selected for simplicity of the computations via COMSOL.

Figs. 2 and 3 depict the magnitudes of the scattered electric fields (that is the difference between the total electric field and the background electric field; the latter is induced in the inclusion-free configuration composed of a three-layered dielectric medium) at all domains. Plane-wave normal illumination is considered for the case of a wet ground and of operating frequency $f=750 \mathrm{MHz}$. In each figure two different sets of contour plots are depicted: the first one corresponds to the case of an absent superstrate and the second to that of an optimal ideal superstrate. The parameters of this optimal superstrate are computed by means of the techniques of Sections II and III. In Fig. 2 the buried inclusion is a PEC strip with different inclination angles while in Fig. 3 a PEC square, rhombus, and circle where all have the same area. Scattering by strip inclusions and inclusions of different crosssections is analyzed in [13], [14], and [15]. More precisely, in [14] the purpose is to hide (or cloak) a PEC strip inside a certain background; here the purpose is the opposite namely to aid the detection of such a strip.

From Figs. 2 and 3 it is evident that the scattered field is significantly enhanced for all scattering problems simulated when the optimal ideal layer is deposited atop the ground. Moreover, we notice that although the optimal parameters of the superstrate layer were calculated via the analysis of the boundary value problem of Section II, involving a linesource primary field and an inclusion of circular shape, these parameters may also offer a significant increase in the scattered field in the problems considered here corresponding to plane wave incidence on buried inclusions of different shapes. 




(a)

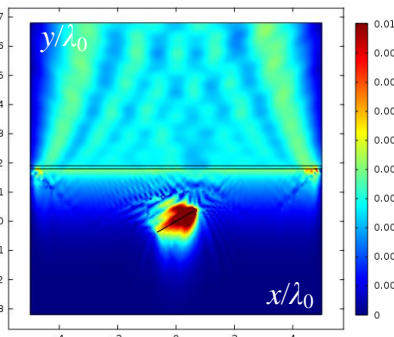

(c)



(e)

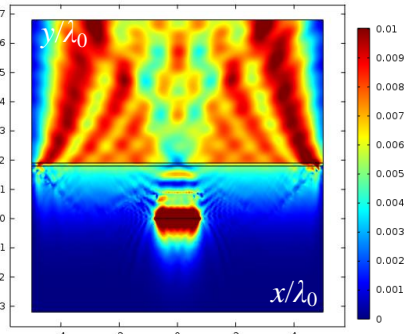

(b)



(d)

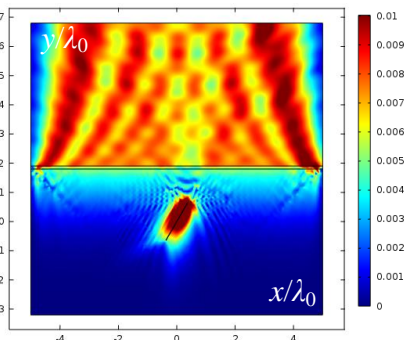

(f)

Fig. 2. Magnitude of the scattered electric field (due to a normally incident plane wave) at all domains for $f=750 \mathrm{MHz}$ and a wet ground and (a) (c), (e) an absent and (b), (d), (f) the optimal ideal superstrate. The buried inclusion is a strip located at $d=1.8 \lambda_{0}$ with width $1.5 \lambda_{0}$ and inclination angle (a), (b) $0^{\circ}$, (c), (d) $30^{\circ}$, and (e), (f) $60^{\circ}$.

\section{CONCLUSIONS}

We considered the problem of detecting a buried inclusion and investigated the significant scattered field enhancement when an optimally determined superstrate is deposited atop the ground. The influence of the shape of the buried inclusion on the amplification of the scattered field was analyzed.

\section{REFERENCES}

[1] A. C. Dubey, and R. L. Barnard (Eds.), Detection and remediation technologies for mines and minelike targets, Proc. SPIE Int. Soc. Opt. Eng. 3079, 1997.

[2] S. Constable and L. J. Srnka, "An introduction to marine controlledsource electromagnetic methods for hydrocarbon exploration," Geophysics, vol. 72, no. 2, pp. WA3WA12, Mar.-April 2007.

[3] H. Huang and I. J. Won, "Automated anomaly picking from broadband electromagnetic data in UXO survey," Geophysics, vol. 68, pp. 18701876 , 2003.

[4] L. Jr. Peters, J. J. Daniels, and J. D. Young, "Ground penetrating radar as a subsurface environmentals sensing tool," Proc. IEEE, vol. 82, pp. 1802 1822, 1994.

[5] D. J. Daniels, Subsurface Penetrating Radar, IEE Press, ch 1, 1996.

[6] L. Crocco and F. Soldovieri, "GPR prospecting in a layered medium via microwave tomography," Ann. Geophys., vol. 46, pp. 559-572, June 2003.

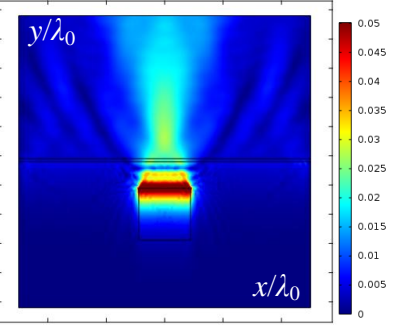

(a)

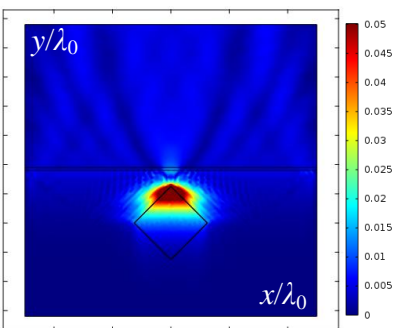

(c)

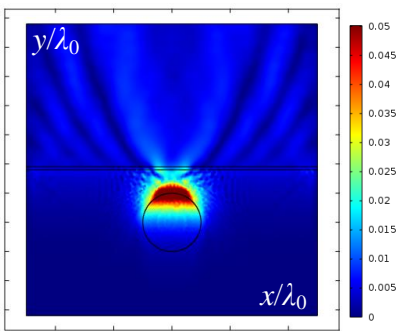

(e)



(b)

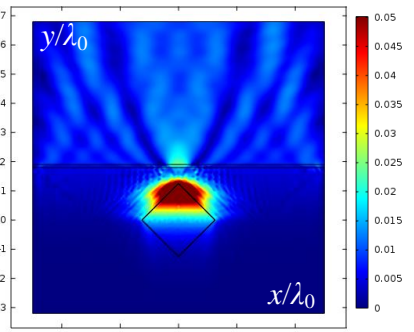

(d)

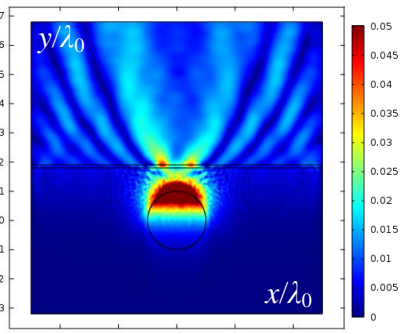

Fig. 3. Magnitude of the scattered electric field (due to a normally incident plane wave) at all domains for $f=750 \mathrm{MHz}$ and a wet ground and (a), (c), (e) an absent and (b), (d), (f) the optimal ideal superstrate. The buried inclusion is (a), (b) a square, (c), (d) a rhombus, and (e), (f) a circle, all of which have the same area $\pi a^{2}=\pi \lambda_{0}^{2}$ and are located at $d=1.8 \lambda_{0}$.

[7] T. J. Cui, W. C. Chew, A. A. Aydiner, and S. Chen, "Inverse Scattering of Two-Dimensional Dielectric Objects Buried in a Lossy Earth Using the Distorted Born Iterative Method," IEEE Trans. Geosc. Rem. Sens., vol. 39, no. 2, pp. 339-346, Feb. 2001.

[8] C. Yu, M. Yuan, Y. Zhang, J. Stang, R. T. George, G. A. Ybarra, W. T. Joines, and Q. H. Liu, "Microwave Imaging in Layered Media: 3-D Image Reconstruction From Experimental Data," IEEE Trans. Antennas Propagat., vol. 58, no. 2, pp. 440-448, Feb. 2010.

[9] C. A. Valagiannopoulos, "Closed-form solution to the scattering of a skew strip field by metallic pin in a slab," Prog. Electrom. Research, vol. 79, pp. $1-21,2008$

[10] C. A. Valagiannopoulos, N. L. Tsitsas, and A. H. Sihvola, "Hiding a bump on a PEC plane by using an isotropic lossless dielectric layer," IEEE Trans. Antennas Propagat., vol. 62, no. 11, pp. 5706-5714, Nov. 2014.

[11] C. A. Valagiannopoulos, N. L. Tsitsas, and A. H. Sihvola, “"Unlocking” the Ground: Increasing the Detectability of Buried Objects by Depositing Passive Superstrates," IEEE Trans. Geosc. Remot. Sens, accepted for publication.

[12] COMSOL Multiphysics. [Online]. Available: http://www.comsol.fi

[13] C. A. Valagiannopoulos, "Electromagnetic Propagation into ParallelPlate Waveguide in the Presence of a Skew Metallic Surface," Electromagnetics, vol. 31, pp. 593-605, 2011.

[14] C. A. Valagiannopoulos and N. L. Tsitsas, "Integral equation analysis of a low-profile receiving planar microstrip antenna with a cloaking superstrate," Radio Science, vol. 47, RS2022, 2012.

[15] C. A. Valagiannopoulos, "Rigorous Solution to Plane Wave Scattering by an Arbitrary-Shaped Particle Embedded into a Cylindrical Cell of Similar Material," Intl. J. Antennas Propagat., vol. 2009, 301461, 2009. 